\title{
Non-destructive Dielectric Measurements and Calibration for Thin Materials Using Waveguide-Coaxial Adaptors
}

\author{
K. Y. You ${ }^{1}$, Z. Abbas ${ }^{2}$, M. F. A. Malek ${ }^{3}$, E. M. Cheng ${ }^{4}$ \\ ${ }^{1}$ Communication Engineering Department, Faculty of Electrical Engineering, Universiti Teknologi Malaysia, \\ 81310 UTM Skudai, Malaysia, e-mail: kyyou@fke.utm.my \\ ${ }^{2}$ Department of Physics, Faculty of Science, Universiti Putra Malaysia, 43400 UPM Serdang, Malaysia, \\ e-mail: za@science.upm.edu.my \\ ${ }^{3}$ School of Electrical Systems Engineering, Universiti Malaysia Perlis, Taman Seberang Jaya Fasa 3, Kuala Perlis, \\ Perlis, Malaysia, e-mail: mfareq@unimap.edu.my \\ ${ }^{4}$ School of Mechatronic Engineering, University Malaysia Perlis, Ulu Pauh Campus, 02600 Arau, Perlis, Malaysia, \\ e-mail: emcheng@unimap.edu.my
}

\begin{abstract}
This paper focuses on the calibration of apertures for rectangular waveguides using open-short-load (OSL) standards and transmission-line (TL) approaches. The reflection coefficients that were measured using both calibration techniques were compared with the coefficients acquired using the thru-reflect-line (TRL) method. In this study, analogous relationships between the results of OSL calibration and TL calibration were identified. In the OSL calibration method, the theoretical, open-standard values are calculated from quasi-static integral models. The proposed TL calibration procedure is a simple, rapid, broadband approach, and its results were validated by using the OSL calibration method and by comparing the results with the calculated integral admittance. The quasi-static integral models were used to convert the measured reflection coefficients to relative permittivities for the infinite samples and the thin, finite samples.
\end{abstract}

Keywords: Relative permittivity, thin materials, rectangular waveguide, reflection coefficient, one-port calibration, admittance aperture.

\section{INTRODUCTION}

耳. OR DIELECTRIC measurements using an open-ended waveguide, a suitable calibration procedure must be applied to the boundary surface between the open-end section of the waveguide and the material being tested [1, $3-10,12-14,18-19]$. Only a few calibration techniques are suitable for open-ended waveguide systems because stray capacitance across the aperture waveguide must be taken into account for open cases. Conventionally, the apertures of rectangular waveguides have been calibrated by using through-reflect-line (TRL) calibration kits $[6,8,10,18]$ or short-short-load (SSL) procedures $[1,7,10]$. Both calibration procedures can give accurate measurements, but they are time-consuming and costly. TRL calibration typically requires two-port measurement instruments $[6,8$, 18 ], and, generally, it is not suitable to use a one-port vector reflectometer. The SSL calibration procedures require time and patience to determine two different location distances of equivalent offset short circuit from the aperture waveguide, which, for a certain frequency, gives the same measured reflection phase as the phase of the shorting plate at the aperture $[1,7]$. For wideband measurements, the SSL calibration procedure is repeated for each frequency point.

In fact, the so-called open-short-load (OSL) calibration technique uses an open-ended coaxial probe $[6,7,9]$. For the open-ended coaxial probe, a liquid, such as water, frequently is used as a load standard for the OSL calibration technique [9], since it does not have any available matchload kit due to the difficultly of constructing a kit that can be connected to an aperture probe. In this work, we attempted to calibrate the aperture of a rectangular waveguide by using the OSL calibration procedures, but the liquid-load standard was replaced by the match-load kit. The open standard values for the calibration were obtained from the calculation of integral admittance. In general, TRL calibration is more accurate than OSL calibration, but the OSL calibration procedure is simpler and less expensive than the TRL and SSL methods. Nevertheless, the accuracy of the OSL calibration is affected significantly by the performance of the waveguides and by the accuracy of the values of the calibration standard. Accuracy levels for the calibration usually are based on the user's requirements. Thus, some recent commercial vector instruments provide several calibration procedures from which the user can choose. In this paper, we also propose another simple calibration model based on the transmission-line principle, which takes into account the measurement noise for open-ended rectangular waveguides. Errors due to systematic noise are caused mainly by discontinuities in the electromagnetic fields at the open end of the waveguide. The transmission-line method is faster than the OSL technique, and it is suitable for fixedwaveguide systems and for measurements that do not require a high level of accuracy. The results of both calibration techniques were analyzed and compared, and they are discussed in detail later. Rectangular waveguide measurements always require a coaxial-rectangular waveguide adaptor to connect the waveguide to the coaxial port. Actually, the waveguide-coaxial adaptor itself can be implemented as a shorter rectangular waveguide. In this work, some waveguide-coaxial adaptors were used to validate the proposed calibration model. The inverse procedures involved the use of a rigorous integral admittance model to predict the relative complex permittivity, $\varepsilon_{r}$, of the thin samples that were tested based on calibrated reflection-coefficient data using the waveguide adaptor. 


\section{COAXIAL-WAVEGUIDE ADAPTORS}

In this study, seven different kinds of right-angle-launch, coaxial-waveguide adaptors were measured. The width of the aperture was $b$, the height of the aperture was $a$, and frequencies in the range of 8.2 to $20 \mathrm{GHz}$ were covered, as shown in Fig.1.a). However, two typical excitation probe configurations, as shown in Figs.1.b) and 1.c), were used by the seven kinds of adaptors. The dimensions and characteristics of the seven kinds of adaptors are listed in Table 1. a) Front view

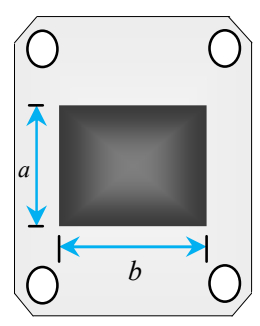

b) Side view

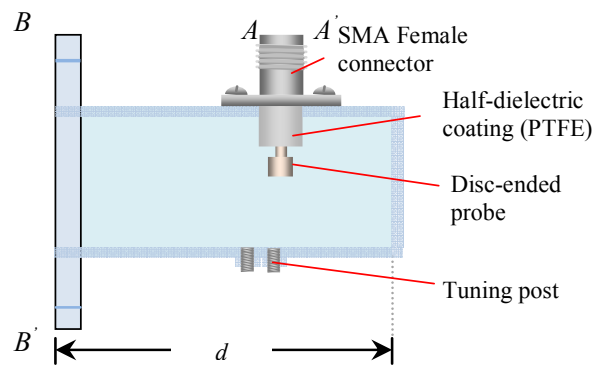

c) Side view

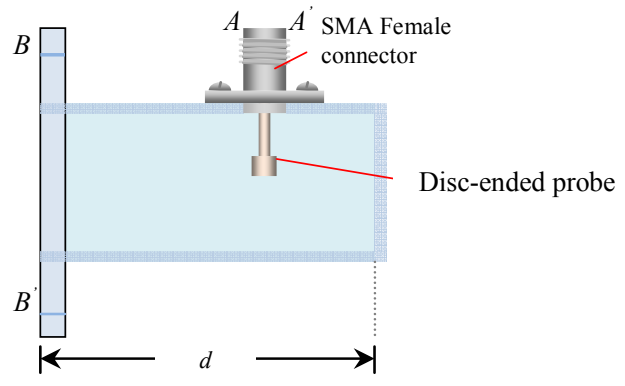

Fig.1. a) Front-view dimensions of the adaptor; b) disc-ended excitation probe (using tuning screws) with a dielectric-coated probe in the adaptor, the length of which is $d$ meter; c) disc-ended excitation probe without a dielectric-coated probe in the adaptor; the length of the probe is $d$ meter.

\section{ONE-PORT CALIBRATIONS}

\section{A. Network error models}

The relationship between the actual reflection coefficient, $\Gamma_{\text {Aperture }}$, at plane $B B$ ' and the measured reflection coefficient, $\Gamma_{\text {Meas }}$, at plane $A A^{\prime}$ (Fig.1.) can be expressed in a bilinear equation as shown below $[6-9,11,12,18]$ :

$$
\Gamma_{\text {Aperture }}=\frac{\Gamma_{\text {Meas }}-c_{2}}{-c_{3} \Gamma_{\text {Meas }}+c_{1}}
$$

The unknown values of the complex calibration coefficients $\left(c_{1}, c_{2}\right.$, and $\left.c_{3}\right)$ in equation (1) were determined by using three calibration standards (open-short-load). Equation (1) can be re-written as a linear expression as shown below:

$$
c_{1} \Gamma_{\text {Aperture }}+c_{2}-\Gamma_{\text {Aperture }} \Gamma_{\text {Meas }} c_{3}=\Gamma_{\text {Meas }}
$$

Let $\Gamma_{A_{-} \text {open }}, \Gamma_{A_{-} \text {short }}$, and $\Gamma_{A_{-} \text {load }}$ represent the known reflection coefficients for the open, short, and load standards that are terminated at the aperture plane $B B$, while, $\Gamma_{M_{-} \text {open }}$,

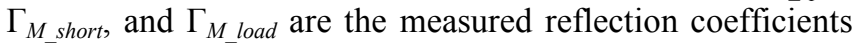
for open, short, and load standards at plane $A A^{\prime}$ '. Finally, three sets of linear equations are created that can be written in matrix form as:

$$
\left[\begin{array}{ccc}
\Gamma_{A_{-} \text {open }} & 1 & -\Gamma_{A_{-} \text {open }} \Gamma_{M_{-} \text {open }} \\
\Gamma_{A_{-} \text {short }} & 1 & -\Gamma_{A_{-} \text {short }} \Gamma_{M_{-} \text {short }} \\
\Gamma_{A_{-} \text {load }} & 1 & -\Gamma_{A_{-} \text {load }} \Gamma_{M_{-} \text {load }}
\end{array}\right]\left[\begin{array}{c}
c_{1} \\
c_{2} \\
c_{3}
\end{array}\right]=\left[\begin{array}{c}
\Gamma_{M_{-} \text {open }} \\
\Gamma_{M_{-} \text {short }} \\
\Gamma_{M_{-} \text {load }}
\end{array}\right]
$$

The values of the open, short, and load standards are given as: $\Gamma_{A_{-} \text {load }}=0+j 0, \Gamma_{A_{-} \text {short }}=-1+j 0$, and $\Gamma_{A_{-} \text {open }}=(1-\tilde{Y}) /(1+\tilde{Y})$. Symbol $\tilde{Y}$ is the normalized input admittance of the aperture for half-free space at plane $B B$, which was computed using the integral, quasi-static admittance model from [3, 4]. The integration in $[3,4]$ was solved by using an $(8 \times 8)$ order, Gaussian, double-integral method. Equation (3) was solved by using a Gaussian elimination routine.

\section{B. Transmission line error models}

The transmission line in the waveguide-coaxial adaptor is coupled between the coaxial line and the rectangular guide line, which converts the propagation wave from coaxial TEM mode into waveguide TE-mode in the operating frequency band. The fringing field effects (stray capacitive effects), which result from mismatches at the transition junction between the coaxial-rectangular waveguide line and the open end of the rectangular guide line, also must be considered from the transmission line. The incident wave from plane $A A^{\prime}$ is transmitted to plane $B B^{\prime}$ by the shifting phase of $\left(k_{c} \delta_{c}+\gamma d+\gamma \delta_{o}\right)$, and it is reflected back to input $A A$ ' with the same shifting phase. Symbols $k_{c} \delta_{c}, \gamma d$, and $\gamma \delta_{o}$ are the phase shifting in the coaxial line, rectangular waveguide, and apparent length of waveguide, respectively. Thus, the aperture reflection coefficient, $\Gamma_{\text {Aperture }}$, at plane $B B^{\prime}$ can be found by the phase delay of $2\left(k_{c} \delta_{c}+\gamma d+\gamma \delta_{o}\right)$ with respect to the measured $\Gamma_{\text {Meas }}$ at plane $A A^{\prime}$. The phase delay is transmitted exponentially from plane $A A^{\prime}$ to plane $B B^{\prime}$, as is evident in (8). In fact, equation (1) is analogous to the transmission line model.

The expression of (1) can be expanded approximately as:

$$
\begin{aligned}
\Gamma_{\text {Aperture }} & \approx\left(\Gamma_{\text {Meas }}-c_{2}\right)\left[\frac{1}{c_{1}}+\frac{c_{3}}{c_{1}^{2}} \Gamma_{\text {Meas }}+\frac{c_{3}^{2}}{c_{1}^{3}} \Gamma_{\text {Meas }}^{2}+\ldots\right] \\
& \approx \frac{\Gamma_{\text {Meas }}}{e_{12} e_{21}}-\frac{e_{11}}{e_{12} e_{21}}-\frac{\Gamma_{\text {Meas }}^{2} e_{22}}{e_{12} e_{21}}+\ldots
\end{aligned}
$$


where $\frac{1}{c_{1}}=\frac{1}{e_{12} e_{21}}+\frac{e_{11} e_{22}}{\left(e_{12} e_{21}\right)^{2}}+\frac{\left(e_{11} e_{22}\right)^{2}}{\left(e_{12} e_{21}\right)^{3}}+\ldots$

The values for the higher-order series terms of (4) are approximated to be zero and assumed to be negligible. The terms $e_{12}$ and $e_{21}$ can be represented by a combination of coaxial and rectangular transmission lines, $\left(k_{c} \delta_{c}+\gamma d+\gamma \delta_{o}\right)$, in which $e_{12}$ and $e_{21}$ were replaced by the transmission phase coefficient, $\exp (-j ø)$ as:

$$
e_{12} e_{21}=\exp \left(-2 j k_{c} \delta_{c}\right) \exp \left[-2 \gamma\left(d+\delta_{o}\right)\right]
$$

a)

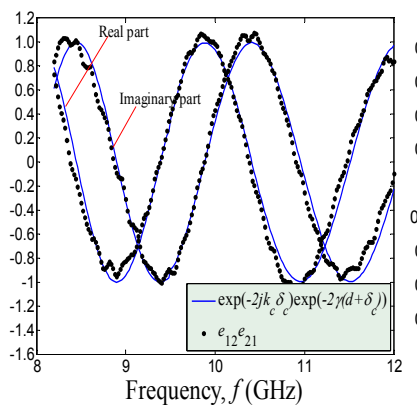

b)

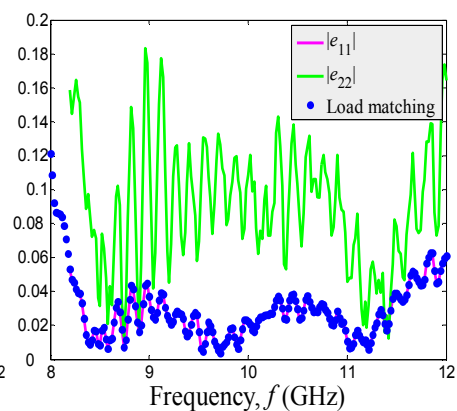

Fig.2. a) The frequency tracking error, $E_{\mathrm{R}}\left(e_{12} e_{21}\right)$ data for Adaptor 1.b) The magnitude of directivity error, $E_{\mathrm{D}}\left(e_{11}\right)$ and the source match error, $E_{\mathrm{S}}\left(e_{22}\right)$ for Adaptor 1

Fig.2.a) shows a comparison of the values of tracking error, $e_{12} e_{21}$, obtained from OSL calibration using Adaptor 1 with the transmission phase coefficient. The values of parameters $d, \delta_{o}$, and $\delta_{c}$ in the transmission phase coefficient are obtained from Table 2 . We found that the two results were in agreement. The directivity error, $e_{11}$, and the source match error, $e_{22}$, are given in Fig.2.b). In fact, the magnitude of $e_{11}$ also can be determined from the match-load measurement, for which the magnitude of the reflection coefficient, $\left|\Gamma_{\text {Meas }}\right|$, at plane $A A^{\prime}$ was measured with the aperture adaptor terminated by a match-load standard. The magnitude of the match-load reflection coefficient approximately overlapped the results of $\left|e_{11}\right|$. For an ideal match load, the reflection coefficient should be zero over the operational frequency. However, it is very difficult to get a perfect match-load standard and a perfect coaxialrectangular adaptor to cover the range of the operational frequency. From Fig.2.b), the directivity error, $\left|e_{11}\right|$, and the match-load measurement error are within an average of 3\% over the X-band frequency by using Adaptor 1, but the magnitudes of the source-match error, $\left|e_{22}\right|$, were within an average of $9 \%$.

In fact, there is a series pairs of forward and reverse reflections and inter-partner interactions at certain positions along the transmission line. Here, we only consider two multiple reflection pairs. $F$ and $R$ are the pair of forward and backward reflections in the outer region of the rectangular waveguide of the aperture, respectively, and $F^{\prime}$ and $R^{\prime}$ are the pair of forward and backward reflections in the inner region near the rectangular waveguide of the aperture. Mutual interaction between the two pairs of reflections can be discretized and expressed as an average of both reflection pairs, i.e., $\left(F+R+F^{\prime}+R^{\prime}\right) / 2$. The second and third terms of (4), which involved $e_{11}$ and $e_{22}$ terms, were assumed to be the forward, $\left(F, F^{\prime}\right)$, and backward, $\left(R, R^{\prime}\right)$, multiple reflections along the transmission line, and the expressions were approximated as:

$$
-\frac{e_{11}}{e_{12} e_{21}}-\frac{\Gamma_{\text {Meas }}^{2} e_{22}}{e_{12} e_{21}} \approx \frac{1}{2}\left(F+F^{\prime}+R+R^{\prime}\right)
$$

where:

$$
\begin{aligned}
& F=\rho^{+} \exp \left[2 \gamma\left(d_{1}-\delta_{1}\right)\right] \\
& F^{\prime}=\rho^{+} \exp \left[2 \gamma\left(d_{1}^{\prime}+\delta_{1}\right)\right] \\
& R=-\rho^{-} \exp \left[2 \gamma\left(d_{1}^{\prime}+\delta_{1}\right)\right] \\
& R^{\prime}=-\rho^{-} \exp \left[2 \gamma\left(d_{1}^{\prime}-\delta_{1}\right)\right]
\end{aligned}
$$

The shift length, $2 \delta_{1}$, between the forward and reflected wave was due to the multiple reflections near the waveguide of the aperture. The amplitude of the forward and backward multiple reflections represented by $\rho^{+}$and $\rho^{-}$was at positions $\left(d_{1}-\delta_{1}\right),\left(d_{1}{ }^{\prime}+\delta_{1}\right),\left(d_{1}{ }^{\prime}-\delta_{1}\right)$, and $\left(d_{1}+\delta_{1}\right)$ near the aperture waveguide, respectively. Finally, the aperture reflection coefficient, $\Gamma_{\text {Aperture }}$, of the sample can be calculated from the measured reflection coefficient, $\Gamma_{\text {Meas }}$, using (8):

$$
\begin{aligned}
\Gamma_{\text {Aperture }}= & \underbrace{\Gamma_{\text {Meas }} \exp \left(2 j k_{c} \delta_{c}\right) \exp \left[2 \gamma\left(d+\delta_{o}\right)\right]}_{\text {Transmission wave in waveguide }} \\
& +\underbrace{\frac{1}{2}\left(F+F^{\prime}+R+R^{\prime}\right)}_{\text {Standing wave in waveguide }}
\end{aligned}
$$

where $\gamma=\alpha+j k_{1}[6]$ and

$$
\begin{gathered}
\alpha=\frac{\sqrt{\pi f \varepsilon_{o} \rho}}{a}\left[\frac{1+(2 b / a)\left(f_{c} / f\right)^{2}}{\sqrt{1+\left(f_{c} / f\right)^{2}}}\right] \\
k_{1}=\sqrt{k_{o}^{2}-(\pi / b)^{2}} \\
k_{c}=\sqrt{k_{o}^{2} \varepsilon_{c}-(\pi / b)^{2}},
\end{gathered}
$$

where symbols $k_{\mathrm{o}}=2 \pi f / c$ and $k_{1}$ are the propagation constants for free space and a rectangular line, respectively; $c$ is the velocity of light in free space; $f$ and $f_{c}$ are the operating frequency and cutoff frequency of the rectangular waveguide, respectively; $\varepsilon_{c}$ is the relative permittivity for 
PTFE, which has the value of $2.06 ; d$ is the actual length of the waveguide adaptor; and $\delta_{c}$ is the tolerance length, which was due to the discontinuous guided wave at the junction between the rectangular waveguide and the coaxial line. Specifically, $\delta_{\mathrm{o}}$ is the dominant tolerance length of the waveguide due to the discontinuous and fringing field near the aperture waveguide. All of the numerical values are listed in Table 2. Phase shift $\left(2 \gamma \delta_{1}\right)$ occurs between the forward reflection, $F$, and the backward reflection, $R$. The dominant transmission wave term in (8) can be represented by Fig.3.a). The forward wave, $F$, and the reflected wave, $R$, were caused mainly by imperfections in the transmission line and a fringing field that occurred near the aperture's rectangular waveguide, as shown in Figs.3.b) and 3.c). The interference from the phase shift between the $F$ and the $R$ along the waveguide causes the measured reflection coefficient to oscillate periodically (systematic noise error) with frequency. Changes in the direction of the oscillation depend on whether the interference is destructive or constructive at a given frequency.

Figs.4.a) and b) show the error between the measured reflection coefficients using Adaptor $1,\left(\Gamma_{\text {quasi-static }}-\Gamma_{\text {Aperture }}\right)$, which does not take into account the effect of the standing wave, with the reflection coefficient obtained from quasistatic model calculation. Clearly, the error, $\left(\Gamma_{\text {quasi-static }}-\right.$ $\left.\Gamma_{\text {Aperture }}\right)$ is in the form of systematic oscillations, which were deduced to have been caused by the standing wave, and the average for the two standing waves, $\operatorname{Re}\left(F+F^{\prime}+R+R^{\prime}\right) / 2$, agrees with the error, as shown in Figs.4.a) and b). Thus, a systematic error in measuring the reflection coefficient can be eliminated by calculating the average effect of the standing wave term in (8).

Table 1. Dimensions and characteristics of waveguide-coaxial adaptors.

\begin{tabular}{|c|c|c|c|c|c|c|c|c|}
\hline $\begin{array}{l}\text { Waveguide } \\
\text { to SMA (F) } \\
\text { Coaxial } \\
\text { Adaptors }\end{array}$ & $\begin{array}{c}\text { Operation } \\
\text { Frequency } \\
\quad(\mathrm{GHz})\end{array}$ & $\begin{array}{l}\text { VSWR } \\
\text { (Max) }\end{array}$ & $\begin{array}{c}\text { Metal } \\
\text { Materials }\end{array}$ & $\begin{array}{c}d \\
(\mathrm{~cm})\end{array}$ & $\begin{array}{c}b \\
(\mathrm{~cm})\end{array}$ & $\begin{array}{c}a \\
(\mathrm{~cm})\end{array}$ & $\begin{array}{c}\text { Tuning } \\
\text { post }\end{array}$ & $\begin{array}{c}\text { Disc-ended, } \\
\text { excitation, coaxial } \\
\text { probe }\end{array}$ \\
\hline Adaptor 1 & $8.2-12.4$ & 1.12 & Brass & 4.25 & 2.286 & 1.016 & yes & $\begin{array}{l}\text { With half-dielectric } \\
\text { coating }\end{array}$ \\
\hline Adaptor 2 & $8.2-12.4$ & 1.12 & Brass & 3.293 & 2.286 & 1.016 & yes & $\begin{array}{l}\text { With half-dielectric } \\
\text { coating }\end{array}$ \\
\hline Adaptor 3 & $8.2-12.4$ & 1.25 & $\begin{array}{c}\mathrm{Al} / \mathrm{Cu} \\
(4 \% \mathrm{Cu})\end{array}$ & 3.42 & 2.286 & 1.016 & no & $\begin{array}{l}\text { Without dielectric } \\
\text { coating }\end{array}$ \\
\hline Adaptor 4 & $10-15$ & 1.25 & $\begin{array}{c}\mathrm{Al} / \mathrm{Cu} \\
(3 \% \mathrm{Cu})\end{array}$ & 2.605 & 1.905 & 0.9525 & no & $\begin{array}{l}\text { Without dielectric } \\
\text { coating }\end{array}$ \\
\hline Adaptor 5 & $11.9-18$ & 1.12 & Brass & 2.53 & 1.5799 & 0.7899 & yes & $\begin{array}{l}\text { With half-dielectric } \\
\text { coating }\end{array}$ \\
\hline Adaptor 6 & $11.9-18$ & 1.12 & Brass & 4.45 & 1.5799 & 0.7899 & yes & $\begin{array}{l}\text { With half-dielectric } \\
\text { coating }\end{array}$ \\
\hline Adaptor 7 & $15-22$ & 1.25 & $\begin{array}{c}\mathrm{Al} / \mathrm{Cu} \\
(7 \% \mathrm{Al})\end{array}$ & 2.308 & 1.2954 & 0.6477 & no & $\begin{array}{l}\text { Without dielectric } \\
\text { coating }\end{array}$ \\
\hline
\end{tabular}

Table 2. Parameters in (8).

\begin{tabular}{|c|c|c|c|c|c|c|c|c|}
\hline Waveguide & $\begin{array}{c}\delta_{c} \\
( \pm 0.01 \mathrm{~cm})\end{array}$ & $\begin{array}{c}\delta_{o} \\
( \pm 0.01 \mathrm{~cm})\end{array}$ & $\begin{array}{c}\delta_{1} \\
( \pm 0.01 \mathrm{~cm})\end{array}$ & $\begin{array}{c}d_{1} \\
( \pm 0.01 \mathrm{~cm})\end{array}$ & $\begin{array}{c}d_{1}{ }^{\prime} \\
( \pm 0.01 \mathrm{~cm})\end{array}$ & $\rho^{+}$ & $\rho^{-}$ & $\alpha$ \\
\hline Adaptor 1 & 0.28 & 0.85 & 0.25 & $d+0.60$ & $d-0.5$ & 0.05 & 0.05 & 0 \\
\hline Adaptor 2 & 0.12 & 1.33 & 0.40 & $d+0.91$ & $d-0.09$ & 0.05 & 0.05 & 0 \\
\hline Adaptor 3 & 0.12 & 1.25 & 0.30 & $d+1.28$ & $d+0.38$ & 0.04 & 0.04 & $2 \times 10^{-4}$ \\
\hline Adaptor 4 & 0.13 & 1.5 & 0.38 & $d+0.80$ & $d+0.1$ & 0.02 & 0.02 & $6 \times 10^{-4}$ \\
\hline Adaptor 5 & 0.42 & 0.86 & 0.40 & $d+1.27$ & $d+0.97$ & 0.025 & 0.025 & $0.7 \times 10^{-4}$ \\
\hline Adaptor 6 & 0.42 & 0.90 & 0.30 & $d+1.35$ & $d+0.65$ & 0.025 & 0.025 & $0.3 \times 10^{-4}$ \\
\hline Adaptor 7 & 0.04 & 0.60 & 0.40 & $d+1.39$ & $d+0.79$ & 0.03 & 0.03 & $9 \times 10^{-4}$ \\
\hline
\end{tabular}


a)

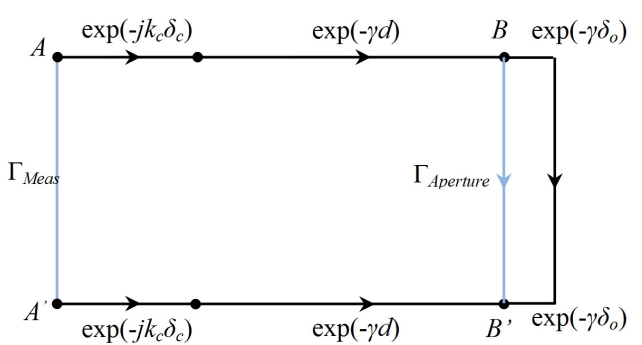

b)

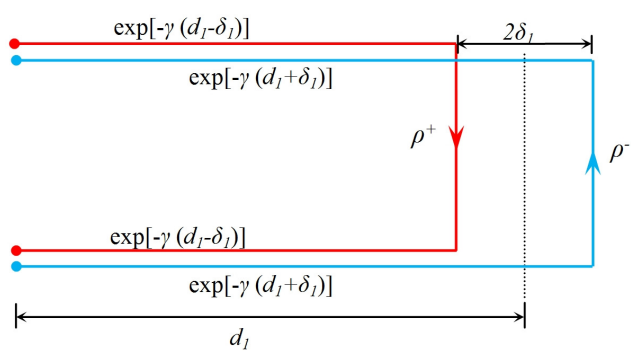

c)

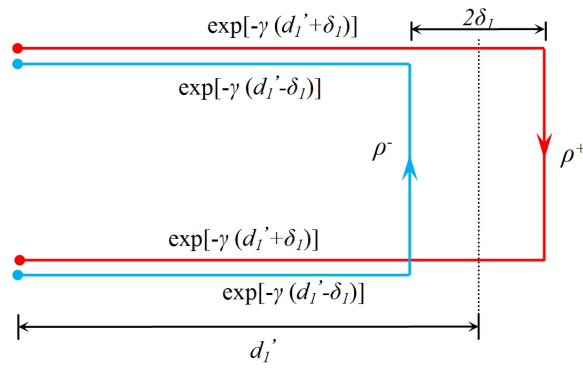

Fig.3. a) Configuration of the main transmission wave along the waveguide; b) first order and c) second order of forward, $F$, and backward, $R$ multiple reflections transmitted along the waveguide.

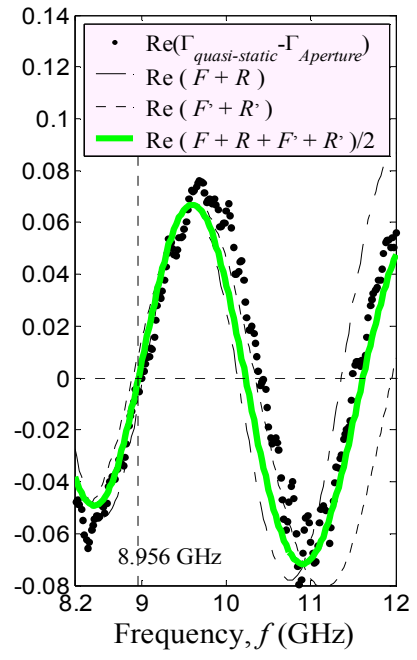

a)

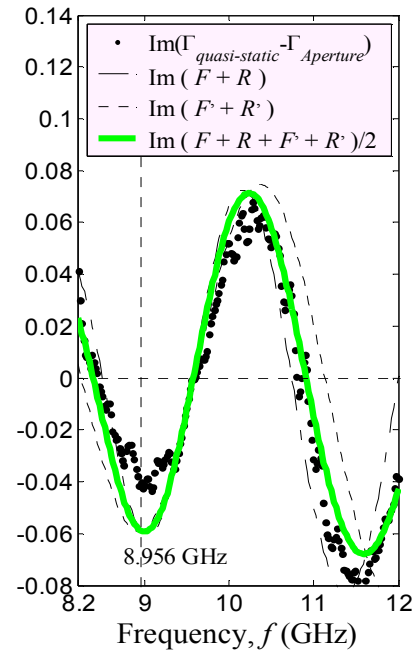

b)
Fig.4. a) Comparison between the average real part of the amplitude of the standing wave, $\operatorname{Re}\left(F+F^{\prime}+R+R^{\prime}\right) / 2$, and the absolute error for real part of measured reflection coefficient, $\operatorname{Re}\left(\Gamma_{\text {quasi-static }}-\Gamma_{\text {Aperture }}\right)$; b) Comparison between the average imaginary part amplitude of standing wave, $\operatorname{Im}\left(F+F^{\prime}+R+R^{\prime}\right) / 2$, and the absolute error for imaginary part of measured reflection coefficient, $\operatorname{Im}\left(\Gamma_{\text {quasi-static }}-\Gamma_{\text {Aperture }}\right)$.

\section{RESULTS AND DISCUSSION}

\section{A. Reflection coefficient and normalized admittance}

Fig.5.a) shows the measured complex reflection coefficient, $\Gamma_{\text {Aperture }}$, by using various kinds of coaxialrectangular adaptors, the ends of which are open to the air. Fig.5.b) shows the measured $\Gamma_{\text {Aperture }}$ for air by considering the standing wave. It is evident that the oscillating noise that occurred in the measured $\Gamma_{\text {Aperture }}$ was removed when the results were compared with the measured results for air in Figs.5.a) and 5.b). The small deviation between experimental results and calculated values may have been caused by cable movements, mismatches between connections in the experimental setup, and instrument errors. The graph line for calibrated reflection coefficient, $\Gamma_{\text {Aperture }}$, versus frequency in Fig.5.b) was improved further by using a filter, which is defined as shown below [17]:

$$
\Gamma_{\mathrm{n}}^{\text {Filter }}=\sigma \Gamma_{\mathrm{n}}^{\text {Data }}+(1-\sigma) \Gamma_{\mathrm{n}-1}^{\text {Filter }}, \quad \text { where } \mathrm{n}=2,3, \ldots \infty
$$

where $\Gamma_{1}^{\text {Data }}=\Gamma_{1}^{\text {Filter }}$, and the values of the initial reflection coefficient, $\Gamma_{1}^{\text {Data }}$, were obtained from measurement data at the lowest frequency points; $n$ is the number of data points; and $\sigma$ is the value of the filter coefficient, which must be in the range of $0<\sigma<1$. The value of $\sigma$ for the filter line in this study was considered to be 0.9 . After being filtered, the reflection results were replotted in Fig.5.c). The transmission model used for correcting the waveguide aperture also was re-examined by using samples of finite thickness. Table 2. shows the parameters used in the transmission calibration.

Equation (8) may be applied for adaptors with different qualities and frequency bands. Figs.6. and 7. compare the results of the measurements and the quasi-static models (12) for paper and the propan-1-ol liquid layer backed by a metallic plate with different thicknesses at $10 \mathrm{GHz}$. The finite thicknesses were measured by using Adaptor 1 and Adaptor 2, respectively, attached to $20 \mathrm{~cm}$ of straight WR 90 rectangular waveguide in order to demonstrate the reliability of the calibration of the transmission line. For the propan-1-ol liquid measurement, the waveguide aperture was attached to one layer of plastic to prevent the liquid sample from entering the interior region of the waveguide. Image theory [13-16] was used to derive the quasi-static admittance model equation (12) as:

$$
\begin{aligned}
\tilde{Y}_{\text {Quasi-Static }}= & \underbrace{\frac{j 8 b}{k_{1} a} \int_{0}^{a} \int_{0}^{b} \chi \frac{e^{-j k_{2} \sqrt{x^{2}+y^{2}}}}{\sqrt{x^{2}+y^{2}}} d x d y \text { Space }}_{\text {Infinite }} d y \text { Medium } \\
& +\underbrace{\frac{j 16 b}{k_{1} a} \sum_{n=1}^{\infty} K^{n} \int_{0}^{a} \int_{0}^{b} \chi \frac{e^{-j k_{2} \sqrt{x^{2}+y^{2}+4 n^{2} h^{2}}}}{\sqrt{x^{2}+y^{2}+4 n^{2} h^{2}}} d x d y}_{\text {Finite }}
\end{aligned}
$$


The $\chi$ parameters in (12) are given as:

$$
\chi=(a-x)\left[D_{1}(b-y) \cos \left(\frac{\pi y}{b}\right)+D_{2} \sin \left(\frac{\pi y}{b}\right)\right]
$$

and

$$
D_{1}=\frac{1}{b^{2}}\left[\frac{k_{2}^{2}}{4 \pi}-\frac{\pi}{4 b^{2}}\right], \quad D_{2}=\frac{1}{\pi b}\left[\frac{k_{2}^{2}}{4 \pi}+\frac{\pi}{4 b^{2}}\right]
$$

where $k_{1}$ and $k_{2}=k_{o} \sqrt{\varepsilon_{r 1}}$ are the propagation constants for the rectangular waveguide line and the external medium with a finite thickness $h$, respectively. In (12), $K$ is the coefficient of the image, given in the form:

$$
K=\frac{\varepsilon_{r 2}-\varepsilon_{r 1}}{\varepsilon_{r 2}+\varepsilon_{r 1}}
$$

where $\varepsilon_{r 2}$ and $\varepsilon_{r 1}$ are the relative permittivities for the first- and second-layer samples, respectively. $K$ equals unity if the thin sample being tested is backed by a metallic plate, because the relative permittivity, $\varepsilon_{r 2}$, approaches infinity, and $K=1$ for a metallic plate. Equation (12) was solved by using an $8 \times 8$-point Gaussian-Legendre integration method. The first term in (12) is exactly equal to the aperture admittance for an infinite half-space medium [3-4], while the second term corresponds to the finite thickness of the material. The measured aperture admittances, $\tilde{Y}_{\text {Aperture }}$, in Figs.6., 7., and 8. were calculated from the measured reflection coefficient, $\Gamma_{\text {Aperture }}$, using the following relationship [1]:

$$
\tilde{Y}_{\text {Aperture }}=\frac{1-\Gamma_{\text {Aperture }}}{1+\Gamma_{\text {Aperture }}}=\frac{G(0)}{Y_{o}}+j \frac{B(0)}{Y_{o}} \text {, }
$$

where $Y_{o}=\sqrt{\varepsilon_{o} / \mu_{o}}$ is the characteristic admittance of the rectangular waveguide; $\varepsilon_{o}$ and $\mu_{o}$ are the permittivity $\left(8.85418782 \times 10^{-12} \mathrm{~F} / \mathrm{m}\right)$ and the permeability $\left(4 \pi \times 10^{-7}\right.$ $\mathrm{H} / \mathrm{m})$ of free space, respectively. The real part, $G(0) / Y_{0}$, and the imaginary part, $B(0) / Y_{\mathrm{o}}$, are called normalized conductance and susceptance, respectively.

The relative complex permittivity, $\varepsilon_{r}$, of the propan-1-ol liquid in the admittance calculations was obtained from the Debye model found in the previous work with the following parameters: $\varepsilon_{s}=20.4, \varepsilon_{\infty}=3.8$, and $\tau=321$ ps for propan-1ol [20], while the value of $\varepsilon_{r}=2.3-j 0.1$ for paper at 10 $\mathrm{GHz}$ was obtained from measurements by using the Agilent dielectric probe. Good agreement can be clearly seen between the calibrated, measured data and the results of the calculations using (12) for the 10 -series terms. The calculated values were found to be in good agreement with measured data, especially for very thin materials $(\leq 1 \mathrm{~mm})$. In Figs.6. and 7., the deviation between the calculated and measured normalized conductance, $G(0) / Y_{o}$, and the susceptance, $B(0) / Y_{o}$, for paper and propan-1-ol liquid could be due to the uncertainties of the relative permittivity values and the difficulty of environmental control for the measurements, since the propan-1-ol measurement was done in a 500-ml beaker, and the uncertainty of the thickness measurement was $\pm 0.1 \mathrm{~mm}$.
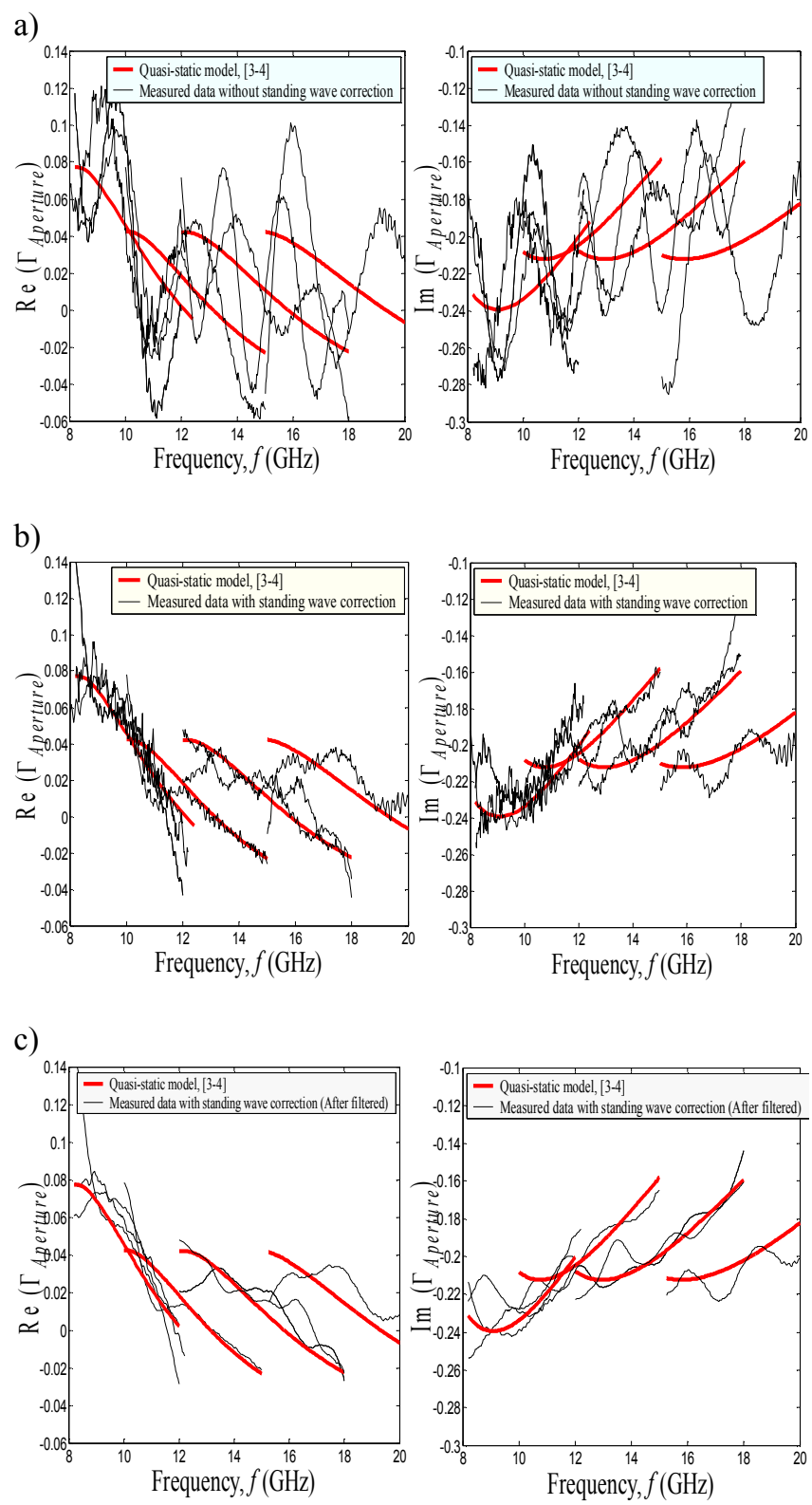

Fig.5. Aperture reflection coefficient at the open-end of the coaxial rectangular adaptors: a) without considering the effects of the standing wave; b) considering the effects of the standing wave; c) considering filtering and the effects of the standing wave. 

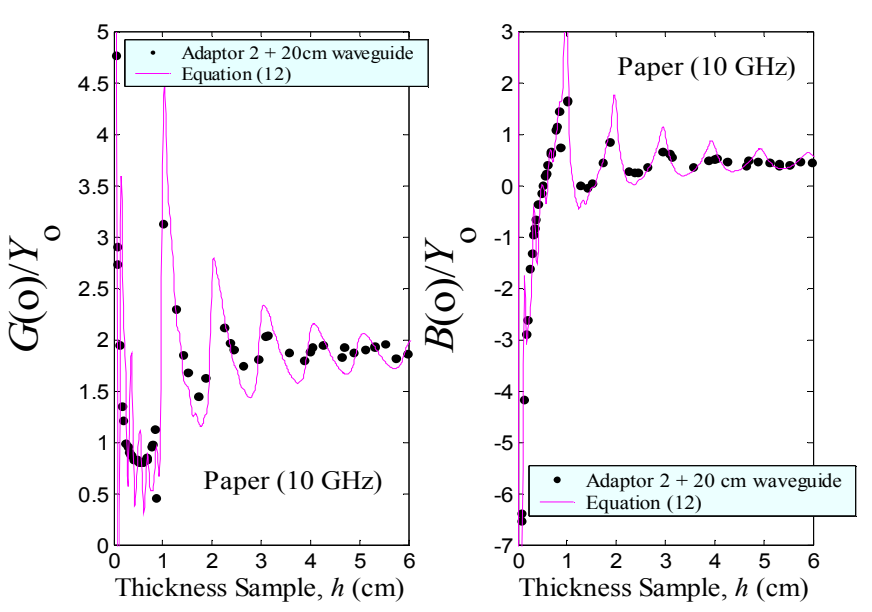

Fig.6. Variation in normalized conductance, $G(0) / Y_{o}$, and normalized susceptance, $B(0) / Y_{o}$, for paper thickness backed by a metallic plate at $10 \mathrm{GHz}$ using Adaptor 2 and a $20 \mathrm{~cm}$ rectangular waveguide.
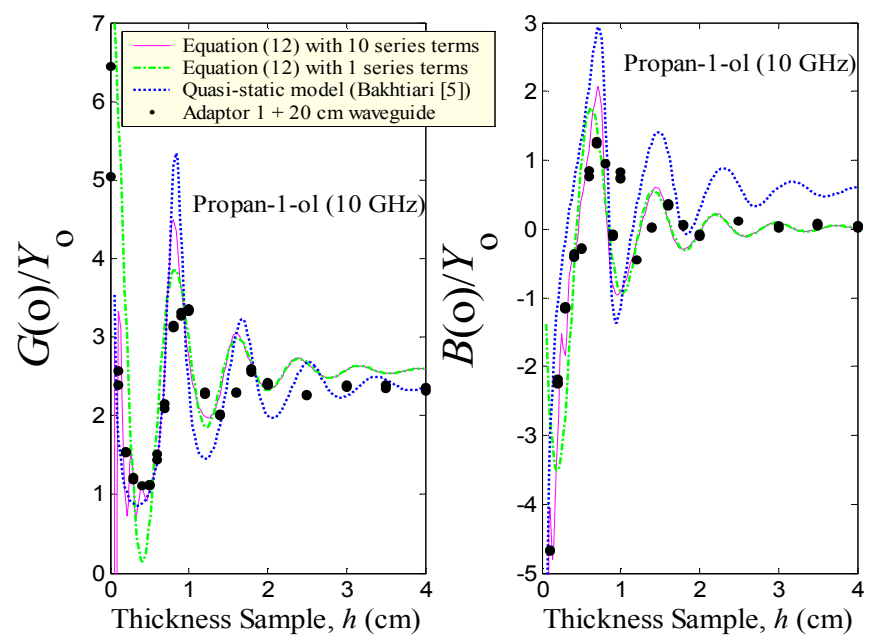

Fig.7. Variation in normalized conductance, $G(0) / Y_{o}$, and normalized susceptance, $B(0) / Y_{o}$, for propan-1-ol thickness backed by a metallic plate at $10 \mathrm{GHz}$ using Adaptor 1 and a $20 \mathrm{~cm}$ rectangular waveguide.
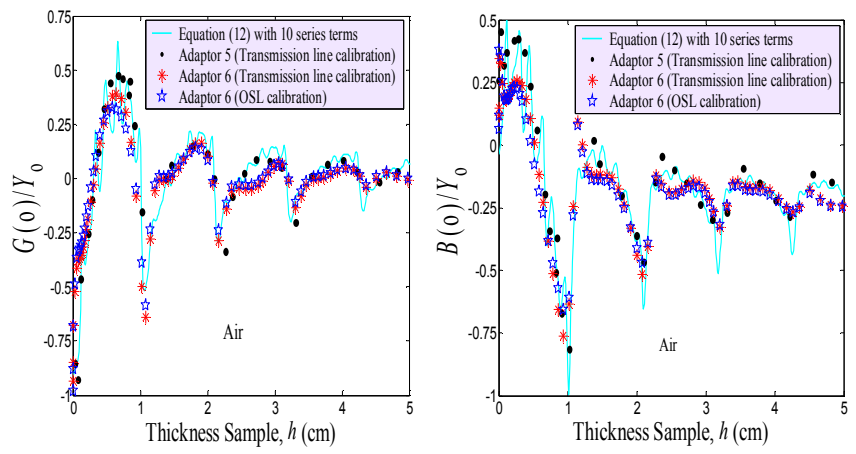

Fig.8. Variation in normalized conductance, $G(0) / Y_{o}$, and normalized susceptance, $B(0) / Y_{o}$, for air thickness backed by a metallic plate at $14 \mathrm{GHz}$ (Ku-band coaxial-rectangular adaptors).

\section{$B$. Inverse relative complex permittivity}

For inverse solutions, first, the measured reflection coefficient, $\Gamma_{\text {Aperture }}$, is transferred to normalized admittance, $\tilde{Y}_{\text {Aperture }}$, via (14). The predicted values of dielectric constant, $\varepsilon_{r}^{\prime}$, are obtained by minimizing the difference between the measured normalized admittance, $\tilde{Y}_{\text {Aperture, }}$ and the quasistatic model, $\tilde{Y}_{\text {Quasi-Static }}$, by referring to the trial function, $\xi$ :

$$
\xi=\left\{\begin{array}{l}
{\left[\operatorname{Re}\left(\tilde{Y}_{\text {Aperture }}\right)-\operatorname{Re}\left(\tilde{Y}_{\text {Quasi-Static }}\right)\right]} \\
+\left[\operatorname{Im}\left(\tilde{Y}_{\text {Aperture }}\right)-\operatorname{Im}\left(\tilde{Y}_{\text {Quasi-Static }}\right)\right]
\end{array}\right\}
$$

The finding zero routine was performed using the MATLAB fzero command in MATLAB. The single, initial, approximate value was selected to be 2 . The main steps of the work were performed using the features of MATLAB, and they are shown in Fig.9.

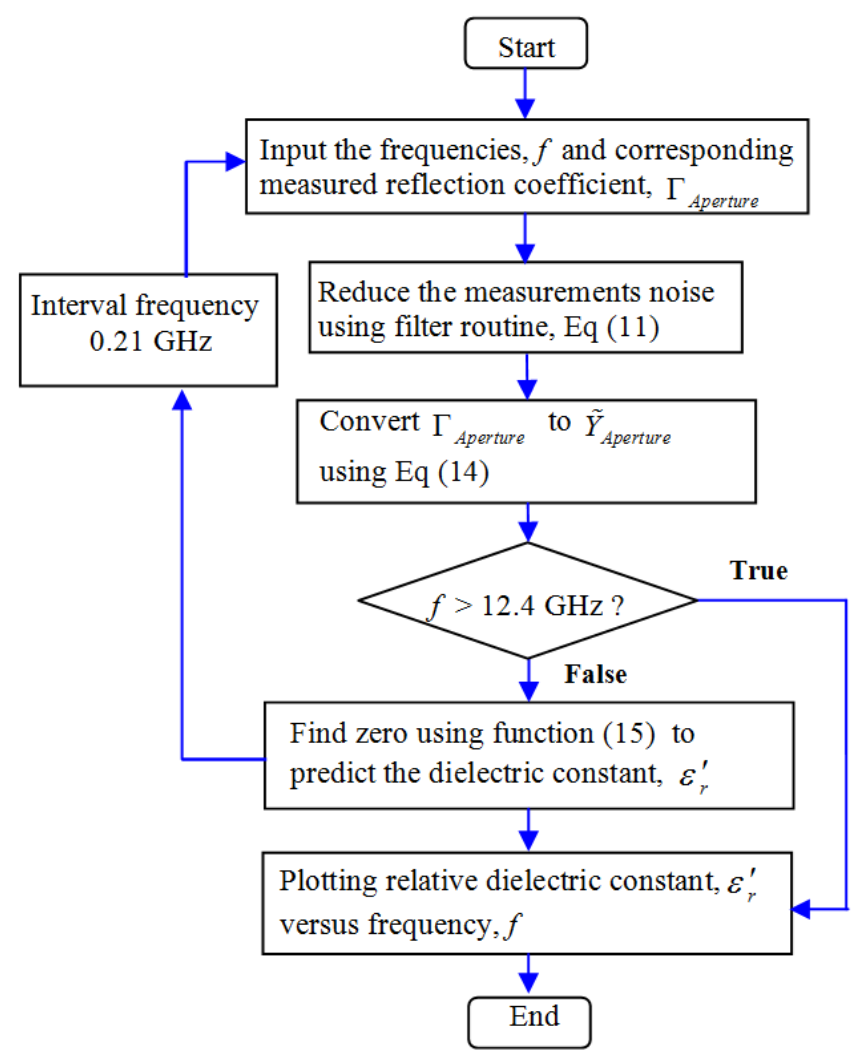

Fig.9. Flow chart of inverse solutions.

Fig.10.a) shows the inversion of the relative dielectric constant, $\varepsilon_{r}^{\prime}$, of air from the measured reflection coefficient, $\Gamma_{\text {Aperture }},\left[\right.$ reflection coefficient, $\Gamma_{\text {Aperture }}$, data obtained from Fig.5.c)] using Adaptor 1. Simultaneously, the deviation between the $\tilde{Y}_{\text {Aperture }}$ and the $\tilde{Y}_{\text {Quasi-Static }}$ from (15) also was plotted in Fig.10.a).

The values of $\Gamma_{\text {Aperture }}$ in the optimization were obtained from the transmission line (TL) calibration. Clearly, the accuracy of predicted values of $\varepsilon_{r}^{\prime}$ is directly dependent on the deviation of (15). The maximum residuals of equation 
(15) for air were nearly \pm 0.04 , which resulted in $3 \%$ of relative error in the predicted dielectric constant of air. Fig.10.b) shows the predicted $\varepsilon_{r}^{\prime}$ for various thicknesses of paper at $10 \mathrm{GHz}$ [normalized input admittance, $\tilde{Y}_{\text {Aperture }}$ data from Fig.6.]. We found it to be very difficult to obtain accurate values of $\varepsilon_{r}^{\prime}$ when the thickness of the paper was less than $0.5 \mathrm{~cm}$. This was due to the high uncertainty that often occurs in small-scale measurements and due to the fact that the scattering of the calculated normalized admittance was high for low values of thickness, $h$, as shown in Fig.6. The number, $n$, of the series term in (12) should be appropriately selected so that the scattering in the calculation of (12) can be reduced and, at the same time, the calculated values are about the same as the measurement data. In this work, 10 series terms were used in equation (12).

a)

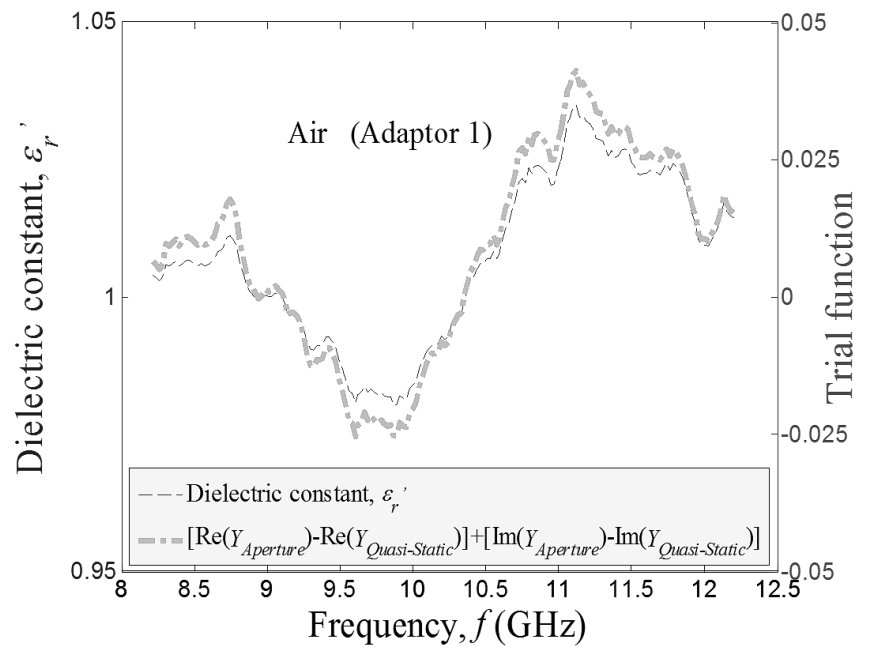

b)

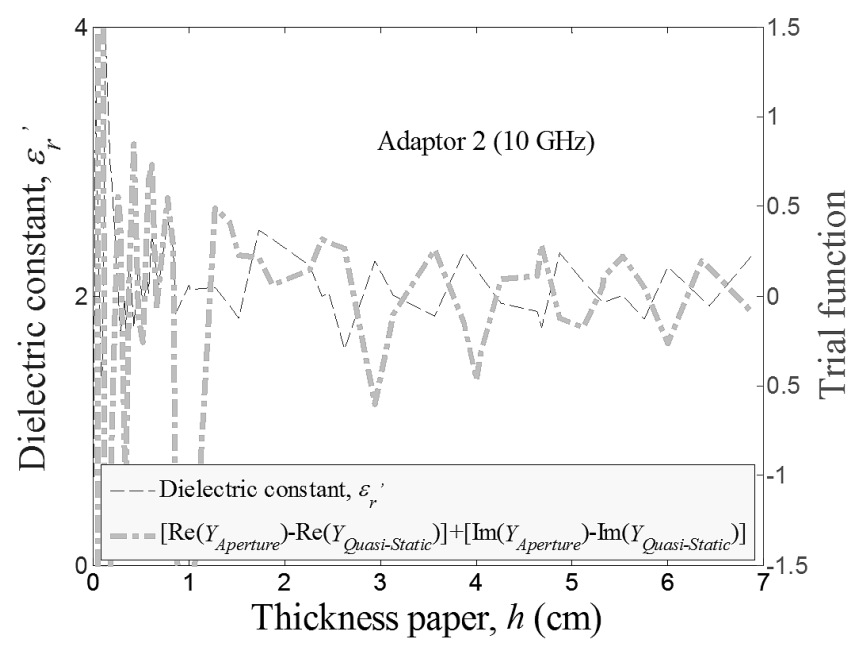

Fig.10. a) Predicted dielectric constant, $\varepsilon_{r}^{\prime}$, of air and its deviation values of trial function (15) using Adaptor 1 for X-band frequencies; b) Predicted dielectric constant, $\varepsilon_{r}^{\prime}$, for various thicknesses, $h$, of paper and its deviation values of trial function (15) using Adaptor 2 at $10 \mathrm{GHz}$.

\section{CONCLUSION}

The open-short-load (OSL) calibration process and the transmission line (TL) calibration process were conducted using seven types of commercial coaxial-waveguide adaptors covering an operating frequency range from 8.2 to $20 \mathrm{GHz}$. The calibration techniques were validated by comparing them with quasi-static admittance solutions. The calibrated results were found to be in good agreement with the measured data over the operational range of frequencies. In this work, the relative dielectric constant, $\varepsilon_{r}^{\prime}$ was predicted from the measurement via a quasi-static admittance model, thereby significantly reducing the problems associated with the inversion accuracy when the open-circuit OSL calibration also was calculated from the quasi-static admittance. Equation (12) was examined with practical cases of one layer of composite material backed by a metallic plate. In fact, equation (12) can be used to predict the dielectric properties of samples that have finite thicknesses and to estimate the equivalent thicknesses of samples of a given dielectric.

\section{ACKNOWLEDGMENT}

This study was supported by a Research University Grant (GUP) from Universiti Teknologi Malaysia under project number Q.J130000.2523.04H77.

\section{REFERENCES}

[1] Chang, C.W., Chen, K.M., Qian, J. (1997). Nondestructive determination of electromagnetic parameters of dielectric materials at X-band frequencies using a waveguide probe system. IEEE Transactions on Instrumentation and Measurement, 46 (5), 1084-1092.

[2] Kim, J.H., Enkhbayar, B., Bang, J.H., Ahn, B.C. (2010). New formulas for the reflection coefficient of an open-ended rectangular waveguide radiating into air including the effect of wall thickness or flange. Progress in Electromagnetics Research M, 12, 143153.

[3] Compton, R.T., Jr. (1963). The aperture admittance of a rectangular waveguide radiating into a lossy halfspace. Technical Report 1691-1, Ohio State University, Columbus, Ohio.

[4] Ganchev, S.I., Bakhtiari, S., Zoughi, R. (1992). A novel numerical technique for dielectric measurement of generally lossy dielectrics. IEEE Transactions on Instrumentation and Measurement, 41 (3), 361-365.

[5] Bakhtiari, S., Ganchev, S.I., Zoughi, R. (1993). Openended rectangular waveguide for nondestructive thickness measurement and variation detection of lossy dielectric slabs backed by a conducting plate. IEEE Transactions on Instrumentation and Measurement, 42 (1), 19-24.

[6] Agilent Technologies. (2010). Specifying Calibration Standards and Kits for Agilent Vector Network Analyzers. Application Note 1287-11. USA: Agilent Technologies, Inc. 
[7] Da Silva, E.F., McPhun, M.K. (1978). Calibration techniques for one-port measurement. Microwave Journal, 21 (6), 97-100.

[8] Fitzpatrick, J. (1978). Error models for systems measurement. Microwave Journal, 21 (5), 63-66.

[9] Kraszewski, A., Stuchly, M.A., Stuchly, S.S. (1983). ANA calibration method for measurements of dielectric properties. IEEE Transactions on Instrumentation and Measurement, 32 (2), 385-386.

[10] Wang, Y., Afsar, M.N. (2005). A calibration technique for measurements of reflection from an open-ended waveguide. In IEEE Antennas and Propagation Society International Symposium, 3-8 July 2005. IEEE, Vol. 3A, 392-395.

[11] Ghannouchi, F.M., Mohammadi, A. (2009). The SixPort Technique with Microwave and Wireless Applications. Boston: Artech House.

[12] You, K.Y., Abbas, Z. (2008). Analytical and numerical analysis of fringing field at aperture openended waveguides. In Second Asia International Conference on Modelling and Simulation (AICMS 08), 13-15 May 2008. IEEE, 277-282.

[13] Anderson, L.S., Gajda, G.B., Stuchly, S.S. (1986). Analysis of an open-ended coaxial line sensor in layered dielectric. IEEE Transactions on Instrumentation and Measurement, 35, 13-18.

[14] Fan, S., Staebell, K., Misra, D. (1990). Static analysis of an open-ended coaxial line terminated by layered media. IEEE Transactions on Instrumentation and Measurement, 39 (2), 435-437.

[15] Galejs, J. (1969). Antennas in Inhomogeneous Media. Oxford: Pergammon.

[16] Siggins, A.F., Gunning, J., Josh, M. (2011). A hybrid waveguide cell for the dielectric properties of reservoir rocks. Measurement Science and Technology, 22, 1-9.

[17] Manassah, J.T. (2006). Elementary Mathematical and Computational Tools for Electrical and Computer Engineers Using MATLAB, $2^{\text {nd }}$ ed. New York: CRC Press.

[18] Challa, R.K., Kajfez, D., Gladden, J.R., Elsherbeni, A.Z. (2008). Permittivity measurement with a nonstandard waveguide by using TRL calibration and fractional linear data fitting. Progress in Electromagnetics Research B, 2, 1-13.
[19] Goňa, S., Křesálek, V. (2009). Derivation of equivalent material models for composite laminated materials. RadioEngineering, 18 (3), 272-284.

[20] Grant, J.P., Clarke, R.N., Symm, G.T., Spyrou, N.M. (1989). A critical study of the open-ended coaxial line sensor technique for RF and microwave complex permittivity measurements. Journal of Physics E: Scientific Instruments, 22 (9), 757-770.

[21] Serizawa, H., Hongo, K. (2005). Radiation for a flanged rectangular waveguide. IEEE Transactions on Antennas and Propagation, 53 (12), 3953-3962.

[22] Yoshitomi, K., Sharobim, H.R. (1994). Radiation from a rectangular waveguide with a lossy flange. IEEE Transactions on Antennas and Propagation, 42 (10), 1398-1403.

[23] Bodnar, D.G., Paris, D.T. (1970). New variational principle in electromagnetics. IEEE Transactions on Antennas and Propagation, 18 (2), 216-223.

[24] Baudrand, H., Tao, J., Atechian, J. (1988). Study of radiation properties of open-ended rectangular waveguides. IEEE Transactions on Antennas and Propagation, 36 (8), 1071-1077.

[25] Ghasr, M.T., Devin, S., Zoughi, R. (2009). Multimodal solution for a waveguide radiating into multi-layered structures-dielectric property and thickness evaluation. IEEE Transactions on Instrumentation and Measurement, 58 (5), 1505-1513.

[26] Jundi, A.J., Qaddoumi, N.N. (2012). Near-field microwave model of multi-layered structures illuminated by open-ended rectangular waveguides. IET Microwaves, Antennas \& Propagation, 6 (1), 100107.

[27] You, K.Y., Abbas, Z. (2011). Transmission wave modelling and calibration in cavity of open-ended rectangular waveguide. In $2^{\text {nd }}$ Annual Passive $R F$ and Microwave Components Seminar, 30 March 2011. IEEE, 79-80.
Received February 2, 2013. Accepted January 23, 2014. 